\title{
Performance Modelling of Pipelined Circuit Switching in Torus with Hot Spot Traffic*
}

\author{
F. Safaei ${ }^{1}$, A. Khonsari ${ }^{1}$, M. Fathy ${ }^{2}$, and M. Ould-Khaoua ${ }^{3}$ \\ ${ }^{1}$ Institute for Studies in Theoretical Physics and Mathematics (I.P.M.) \\ ${ }^{2}$ Dept. of Computer Engineering, Iran Univ. of Science and Technology, Tehran, Iran \\ ${ }^{3}$ Dept. of Computing Science, University of Glasgow, UK \\ \{safaei, ak\}@ipm.ir, mahfathy@iust.ac.ir, mohamed@dcs.gla.ac.uk
}

\begin{abstract}
This paper proposes a new analytical model of PCS in torus in the presence of hot spot traffic pattern. Results from simulation experiments show close agreement with those predicted by the analytical model.
\end{abstract}

\section{Introduction}

Gaughan and Yalamanchili [1] have proposed PCS that combines aspects of Circuit Switching (CS) and Wormhole Switching. When a message header encounters blocking and cannot progress towards its destination, it releases the last reserved channel by backtracking to the previous node, and then continues its search from that node to find an alternative path. Recent studies [2, 3] have revealed that the performance advantages of adaptive routing are more noticeable when traffic is nonuniform due to the presence of hot spots [4]. This paper proposes a new analytical model of PCS for computing the average message latency in the presence of hot spot traffic in torus.

\section{The Analytical Model}

PCS and the router structure are discussed in detail in $[1,3]$. The model is based on the following assumptions.

i) The traffic model is based on Pfister and Norton approach [4]. In their method, each generated message has a finite probability $\theta$ of being directed to the hot spot node and probability $(1-\theta)$ of being directed to other network nodes. We usually refer to these types of messages as hot spot and regular, respectively.

ii) Nodes generate traffic independently of each other, which follows a Poisson process with a mean arrival rate of $\lambda_{g}$ messages per node per cycle including regular and hot spot fractions, $\theta \lambda_{g}$ and $\left(1-\theta \lambda_{g}\right)$, respectively. Message length is $M$ flits, each of which requires one cycle to cross from one node to the next.

iii) $L$ virtual channels $(L \geq 1)$ are used per physical channel.

\footnotetext{
* This research was in part supported by a grant from I.P.M. (No. CS1384-3-01).
} 
The average message latency is composed of the average network latency, $\bar{T}$, and the average waiting time seen by a message at the source node, $\bar{W}$. However, to capture the effects of virtual channels multiplexing, the average message latency has to be scaled by a factor, $\bar{L}$, representing the average degree of virtual channels multiplexing that takes place at a given physical channel. Therefore, we can write [2]

$$
\text { Latency }=(\bar{T}+\bar{W}) \bar{L} \text { where } \bar{T}=(1-\theta) \overline{T_{r}}+\theta \bar{T}_{\theta}
$$

In the above equation, $\overline{T_{r}}$ and $\overline{T_{\theta}}$ denote the average network latency for regular and hot spot messages, respectively. The average number of hops that a regular message makes across one dimension and across the network, $\bar{k}$ and $\bar{d}_{r}$ respectively, are [3]

$$
\bar{k}=(k-1) / 2 \quad, \quad \bar{d}_{r}=2 \bar{k}
$$

Since each regular message travels, on average, $\bar{d}_{r}$ hops to cross the network, the rate of regular messages received by each channel, $\lambda_{r}$, can be written as

$$
\lambda_{r}=(1-\theta) \lambda_{g} \bar{d}_{r} / 2=(1-\theta) \lambda_{g} \bar{c}_{r} / 4
$$

Where, $\bar{c}_{r}$ is the average time needed to setup a path for a regular $r$-hop header. The number of source nodes for which one of $2 N_{j-1}$ channels can act as intermediate channel to reach the hot spot node is given by [2]

$$
N-\sum_{r=0}^{j-1} N_{r}=\sum_{r=j}^{2(k-1)} N_{r} \quad ; \quad N_{r}=0 \quad \text { if } r>2 k-2, N_{r}= \begin{cases}r+1 & r<k \\ 2 k-r-1 & k \leq r \leq 2 k-2\end{cases}
$$

The overall traffic rate, on the channel located $j$ hops from the hot spot node, is

$$
\lambda_{j}=\lambda_{r}+\lambda_{\theta_{j}} \quad \text { where } \quad \lambda_{\theta_{j}}=\left(\theta \lambda_{g} \sum_{r=j}^{2(k-1)} N_{r}\right) /\left(2 N_{j-1}\right)
$$

In the above equation, each node generates, on average, $\theta \lambda_{g}$ hot spot messages in a cycle, and $\lambda_{\theta_{j}}$ is the rate of hot spot traffic on a channel located $j$ hops away from hot spot node. In PCS, the network latency of an $r$-hop regular message can be written as

$$
\bar{T}_{r}=M+r+\bar{c}_{r}
$$

The latency seen by a hot spot message that is $j$ hops a way from the hot spot node is

$$
\bar{T}_{\theta_{j}}=M+j+\bar{c}_{\theta}
$$

Where, $\bar{c}_{\theta}$ is the average time needed to setup a path for a hot spot message header. When a regular (or hot spot) message reaches a channel that is $j$ hops away from the hot spot node, the mean service time at the channel, considering both regular and hot spot message with their appropriate weights, can be written as

$$
\bar{T}_{j}=\left(\lambda_{c} / \lambda_{j}\right) \bar{T}_{r}+\left(\lambda_{\theta_{j}} / \lambda_{j}\right) \bar{T}_{\theta_{j}}
$$


Finally, by averaging all possible values of $\bar{c}_{r}$ and $\bar{c}_{\theta}$ yields the overall average time to set up a path, $\bar{c}$, is given by

$$
\bar{c}=(1-\theta) \bar{c}_{r}+\theta \bar{c}_{\theta}
$$

The average network latency seen by a hot spot message can be written as

$$
\bar{T}_{\theta}=\sum_{j=1}^{2(k-1)} p_{\theta_{j}} \bar{T}_{\theta_{j}} \text { where } p_{\theta_{j}}=N_{j} /(N-1)
$$

We model the header behaviour as a Random Walk problem [3]. $\bar{c}_{j}$ which is the average time interval to reach the destination, satisfies the following equation

$$
\bar{c}_{j}=0 \quad \text { if } j=r \quad \text { otherwise } \quad \bar{c}_{j}= \begin{cases}\left(1-p b_{0}\right)\left(c_{1}+1\right)+p b_{0} \bar{c}_{0} & j=0 \\ \left(1-p b_{j}\right) \bar{c}_{j+1}+p b_{j} \bar{c}_{j-1}+1 & 1 \leq j \leq r-1\end{cases}
$$

The average time to setup a path for an $r$-hop regular message and the time is needed to setup a reserved path for hot spot message that is $j$ hops away $(1 \leq j \leq 2(k-1))$ from the hot spot node are given by

$$
\bar{c}_{r}=\bar{c}_{0}+r \quad, \bar{c}_{\theta j}=\bar{c}_{0}+j
$$

The probability of blocking, can therefore written as

$$
\operatorname{pb}_{j}=\operatorname{pass}_{j}^{0} \cdot\left(P_{L}\right)^{2}+\operatorname{pass}_{j}^{1} \cdot P_{L} \quad ; \operatorname{pass}_{j}^{0}=(1-\bar{k})^{2}, \operatorname{pass}_{j}^{1}=2(1-\bar{k}) / \bar{k}
$$

Where $\operatorname{pass}_{j}^{0}$ and $\operatorname{pass}_{j}^{l}$ are the probability that a message has to visit one dimension and the probability that it still has to visit both dimensions, respectively. To determine the average time, $\bar{W}$, that a message sees in the source node before entering into the network, the injection channel is treated as an $M / G / 1$ queue with a mean time waiting of [2]

$$
\bar{W}=\rho \bar{T}\left(1+C_{\bar{T}}^{2}\right) /(2(1-\rho)) \quad, \quad \rho=\lambda_{g} \bar{T} \quad, \quad C_{\bar{T}}^{2}=\sigma_{\bar{T}}^{2} / \bar{T}^{2}
$$

Where ${\sigma_{\bar{T}}}^{2}$ is the variance of the service distribution. The average arrival rate on each virtual channel is $\lambda_{g} / L$ and service time, $\bar{T}$, with an approximated variance $(\bar{T}-M-3 \bar{d}+1)^{2}$ yields the mean waiting time as

$$
\bar{W}=\bar{T}^{2}\left(\lambda_{g} / L\right)\left[1+(\bar{T}-M-3 \bar{d}+1)^{2} / \bar{T}^{2}\right] /\left(2\left[1-\bar{T}\left(\lambda_{g} / L\right)\right]\right)
$$

The probability, $P_{l_{j}}$, that $l$ virtual channels are busy at the physical channel that is $j$ hops away from the hot spot node, can be determined as follows 


$$
P_{l_{j}}=\left(1-\lambda_{j} \overline{T_{j}}\right)\left(\lambda_{j} \overline{T_{j}}\right)^{l} \quad \text { if } \quad 0 \leq l<L \quad \text { otherwise } \quad P_{l_{j}}=\left(\lambda_{j} \overline{T_{j}}\right)^{l} \quad l=L
$$

And the average multiplexing rate through the network is

$$
\bar{L}=\sum_{j=l}^{2(k-1)} p_{\theta_{j}} \bar{L}_{j} \quad \text { where } \quad \bar{L}_{j}=\sum_{l=l}^{L} l^{2} P_{l_{j}} / \sum_{l=l}^{L} l P_{l_{j}}
$$

Fig. 1 shows the latency results predicted by the model against those provided by the simulator for Network size $N=8 \times 8$ torus, Message length is $M=32$ and 64 flits, number of virtual channels $L=6$, and fractions of hot spot traffic is $\theta=0.05$ and 0.2 .
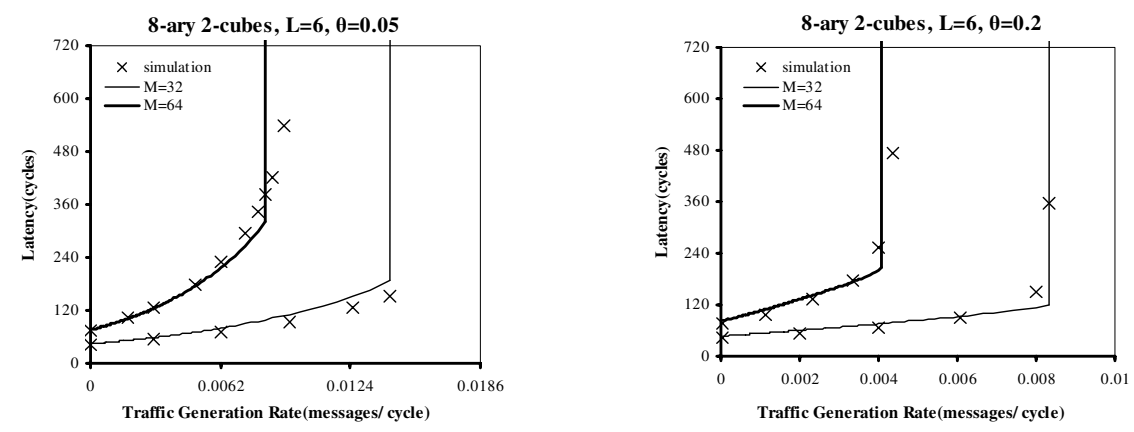

Fig. 1. Average message latency calculated by model vs. simulation

\section{Conclusions}

In this paper we proposed a new analytical model to compute the average message latency of PCS in two-dimensional torus in the presence of hot spot traffic. Simulation experiments have revealed that the results predicted by the model are in good agreement with those obtained through simulations under different working conditions.

\section{References}

1. P.Gaughan, S. Yalamanchili, "A family of fault-tolerant routing protocols for direct multiprocessor networks", IEEE TPDS, 6(5): 482-497, 1995.

2. H. Sarbazi-Azad, L. Mackenzie, M. Ould-Khaoua, "Hot Spot Analysis in Wormhole-routed Tori”, IPCCC 2000. Conference proceeding of the IEEE International, 337-343, 2000.

3. F. Safaei, A.. Khonsari, M. Fathy, M. Ould-Khaoua, "An analytical model of Pipelined Circuit Switching in hypercubes in the presence of hot spot traffic", ICPP 2005 conference proceedindg of the IEEE international, 485-492, June 2005.

4. G.J. Pfister, V.A. Norton, "Hot spot contention and combining in multistage interconnection networks”, IEEE Trans. Computers, 34(10): 943-948, 1985. 